\title{
Performance and Chemical Composition of 18 Nondormant Alfalfa Cultivars at the Lajas Valley ${ }^{1}$
}

\author{
J. Vélez-Santiago, J. A. Arroyo-Aguilú, S. Torres-Rivera and N. \\ Corchado Juarbe ${ }^{2}$
}

\begin{abstract}
Eighteen alfalfa (Medicago sativa) cultivars were evaluated as to dry forage and crude protein yields at the Lajas Research and Development Center; there were 6 cuttings during a 232-day period. Dry forage yields ranged from 4,673 $\mathrm{kg} / \mathrm{ha}$ for Tanverde (lowest) to $11,675 \mathrm{~kg} / \mathrm{ha}$ for Maracay (highest) cultivar. Good yields were also obtained with Florida 66, Moapa, Peruvian, Certified Mesa Sirsa, and Hayden PX-1 cultivars. Stand persistence declined markedly after the fifth harvest due to Phytophthora root rot infestation. Maracay, Florida 66, and Certified Mesa Sirsa showed the best persistence through the sixth cutting. Mean overall values for neutral-detergent fiber, acid-detergent fiber, lignin, cellulose, silica, crude protein, phosphorus, and potassium contents for the 18 cultivars were $34.86,29.13,10.22,17.08,1.42,21.86,0.36$, and $2.08 \%$, respectively. Insects were not a major problem in this study.
\end{abstract}

\section{INTRODUCTION}

Because of the outstanding nutritional properties of alfalfa (Medicago sativa), there is great interest in the introduction and evaluation of new cultivars of this forage for use on the southern coast of Puerto Rico. In the form of pellets or hay, alfalfa would be very useful as a feed ingredient in horse, rabbit, and poultry rations. Also, appealing is associating alfalfa with tropical grasses to increase forage yield and nutritive value during the cool months.

Freyre et al. (2), in 1954, compared the yields of 15 alfalfa cultivars at the Lajas Agricultural Experiment Substation. Among the best ary forage (DF) yielders in four harvest were Peruvian, Uruguay 10, and ArizonaChilean, with 12344, 12061, and $12053 \mathrm{~kg} / \mathrm{ha}$, respectively. They also reported a lack of stand persistence after the fifth harvest because of root rot caused by unidentified organism. In Arizona, Hine et al. (5) reported that this disease, caused by the fungus Phytophthora megasperma, was an important factor in yield decline. A recent preliminary screening trial with 25 nondormant cultivars at the Corozal Agricultural Experiment Substation demonstrated that the best cultivars were Florida 66, Mesa Sirsa LB-1, and Hayden PX-1 (11).

${ }^{1}$ Manuscript submitted to Editorial Board March 12, 1982.

${ }^{2}$ Associate Agronomist, Agricultural Experiment Substation, Corozal, P. R.; Animal Nutritionist, Animal Industry Department, Agricultural Experiment Station, University of Puerto Rico, Río Piedras, P. R.; Research Technician, Agricultural Experiment Substation, Corozal, P. R. an Assistant, Animal Industry Department, Agricultural Experiment Station, University of Puerto Rico, Rio Piedras, P. R. 
The objective of the present study was to determine the adaptability of various alfalfa varieties to conditions on the southern coast of Puerto Rico in terms of forage yields and chemical composition.

\section{MATERIALS AND METHODS}

The trial was conducted at the Lajas Research and Development Center during a 232-day period from January 31, 1978 to September 20, 1978, and involved 18 alfalfa cultivars (table 1). After the initial cutting, these were harvested every 30 days at the $25 \%$ bloom stage on six different occasions.

The soil was a Fraternidad clay (Vertisol), the top $20 \mathrm{~cm}$ layer of which contained $12.5 \mathrm{p} / \mathrm{m}$ of phosphorus (P), $194 \mathrm{p} / \mathrm{m}$ of potassium $(\mathrm{K})$, and gave a $\mathrm{pH}$ reading of approximately $6.3(7)$.

The experimental layout was a partially-balanced incomplete block design with four replicates. Individual plots were $213.36 \mathrm{~cm}$ wide $\times 487.68$ $\mathrm{cm}$ long and consisted of seven plant rows $30.48 \mathrm{~cm}$ apart. Green forage (GF) yields were obtained from the five-center rows only.

Alfalfa seeds were inoculated with Rhizobium meliloti, immediately before hand planting. Aerial irrigation was applied on the following dates: February 1 and 16; March 1, 18, and 23; April 24 and 28; May 20 and 31; June 30; July 7 and 31; August 5 and 7; and September 18. Three hand weedings of the experimental plots were necessary. The only fertilizer used during the course of the experiment was borax, which was applied 4 mo after planting at a rate of $5.6 \mathrm{~kg} / \mathrm{ha}$, because of deficiency symptoms of this mineral.

All cultivars were harvested at a height of approximately $3 \mathrm{~cm}$ from the ground with a Gravely ${ }^{3}$ sickle bar machine, and the forage was immediately weighed. Plot samples were taken, dried at $60^{\circ} \mathrm{C}$, and ground in a Wiley mill to pass through a 1 -mm screen.

Dry matter (DM) content was determined in all samples. For each harvest, samples were composited by replications and each cultivar was subjected to sulphuric acid digestion (8), N, P, and K prior to determination with a Technicon auto analyzer. Crude protein was calculated as $\mathrm{N} \times 6.25$. Representative samples from each cultivar were analyzed for neutral-detergent fiber (NDF), acid-detergent fiber (ADF), lignin (L), cellulose (C), and silica (Si) (3). Neutral-detergent soluble (NDS) content was calculated as the difference between 100 and NDF; and hemicellulose $(\mathrm{H})$ content as the difference between NDF and ADF.

\footnotetext{
${ }^{3}$ Research Trade names in this publication are used only to provide specific information. Mention of a trade name does not constitute a warranty of equipment or materials by the Agricultural Experiment Station of the University of Puerto Rico, nor is this mention a statement of preference over other equipment or materials.
} 
TABLE 1.-Identification of 18 alfalfa cultivars

\begin{tabular}{|c|c|}
\hline Cultivar & Seed Source \\
\hline Maracay & $\begin{array}{l}\text { FAO-United Nations Development Program, Santo } \\
\text { Domingo, Dominican Republic }\end{array}$ \\
\hline Florida 66 & $\begin{array}{l}\text { Agronomy Department, Univ. of Florida, Gainesville, } \\
\text { Florida } 32611\end{array}$ \\
\hline Certified Mesa Sirsa & $\begin{array}{l}\text { Plant Sciences Department, Univ. of Arizona, Tuczon, } \\
\text { Arizona } 85721\end{array}$ \\
\hline Moapa & $\begin{array}{l}\text { FAO-United Nations Development Program, Santo } \\
\text { Domingo, Dominican Republic }\end{array}$ \\
\hline Hayden PX-1 & $\begin{array}{l}\text { Plant Sciences Department, Univ. of Arizona, Tuczon, } \\
\text { Arizona } 85721\end{array}$ \\
\hline Peruvian & $\begin{array}{l}\text { FAO-United Nations Development Program, Santo } \\
\text { Domingo, Dominican Republic }\end{array}$ \\
\hline UC-76-E & $\begin{array}{l}\text { Agric. Sciences Division, Imperial Valley Field Station, } \\
\text { Univ. of California, El Centro, California } 92243\end{array}$ \\
\hline Sonora-70 & $\begin{array}{l}\text { Plant Sciences Department, Univ. of Arizona, Tuczon, } \\
\text { Arizona } 85721\end{array}$ \\
\hline Certified Hayden & $\begin{array}{l}\text { Plant Sciences Department, Univ. of Arizona, Tuczon, } \\
\text { Arizona } 85721\end{array}$ \\
\hline AZ Mexon (scald tol.) & $\begin{array}{l}\text { Plant Sciences Department, Univ, of Arizona, Tuczon, } \\
\text { Arizona } 85721\end{array}$ \\
\hline Mesa Sirsa Lateral Branch & $\begin{array}{l}\text { Plant Sciences Department, Univ. of Arizona, Tuczon, } \\
\text { Arizona } 85721\end{array}$ \\
\hline UC-Salton & $\begin{array}{l}\text { Agric. Sciences Division, Imperial Valley Field Station, } \\
\text { Univ. of California, El Centro, California } 92243\end{array}$ \\
\hline UC-Cargo & $\begin{array}{l}\text { Agric. Sciences Division, Imperial Valley Field Station, } \\
\text { Univ. of California, El Centro, California } 92243\end{array}$ \\
\hline CUF-101 & $\begin{array}{c}\text { Agric. Sciences Division, Imperial Valley Field Station, } \\
\text { Univ. of California, El Centro, California } 92243\end{array}$ \\
\hline WL-311 & $\begin{array}{l}\text { Penington Seed, Inc., P. O. Box 290, Madison, Georgia } \\
\quad 30650\end{array}$ \\
\hline Liberty (NCW-20) & $\begin{array}{l}\text { USDA ARS Southern Region, North Carolina State } \\
\text { Univ., Raleigh, North Carolina } 27607\end{array}$ \\
\hline Thor & $\begin{array}{l}\text { Northrup King Co., P. O. Box 959, Minneapolis, Min- } \\
\text { nesota } 55440\end{array}$ \\
\hline Tanverde & $\begin{array}{l}\text { USDA Regional Plant Introduction Station, Experi- } \\
\text { ment, Georgia } 30212\end{array}$ \\
\hline
\end{tabular}

Means for GF and DF yields and DM content were compared by Duncan's (1) multiple range test (10).

\section{RESULTS AND DISCUSSION}

The monthly rainfall pattern during the 232-day period was as follows: $55.9 \mathrm{~mm}$ (February; $106.9 \mathrm{~mm}$ (March); $82.5 \mathrm{~mm}$ (April); $25.4 \mathrm{~mm}$ (May); $25.0 \mathrm{~mm}$ (June); $59.2 \mathrm{~mm}$ (July); $278.3 \mathrm{~mm}$ (August); and $9.4 \mathrm{~mm}$ (September 1-20), for a total of $642.6 \mathrm{~mm}$. The August rainfall repre- 
sented $43.3 \%$ of the total. The 15 aerial irrigations were equivalent to $190 \mathrm{~mm}$.

Uniform stands were obtained initially for all cultivars except for Tanverde. Tanverde stands in each replicate were approximately $75 \%$ as heavily populated as the rest of the cultivars.

Table 2 shows the total GF, DF, and CP yields and DM content. The highest GF yielders were Maracay, Florida 66, and Certified Mesa Sirsa cultivars, while the lowest were WL-311, Liberty (NCW-20), Thor, and

TABLE 2.-Total green forage, dry forage and crude protein yields and mean dry matter content of 18 alfalfa cultivars during a 232-day period

\begin{tabular}{|c|c|c|c|c|}
\hline Cultivar & $\begin{array}{l}\text { Green } \\
\text { forage } \\
\text { yield }\end{array}$ & $\begin{array}{c}\text { Dry } \\
\text { matter } \\
\text { content }\end{array}$ & $\begin{array}{c}\text { Dry } \\
\text { forage } \\
\text { yield }\end{array}$ & $\begin{array}{l}\text { Crude } \\
\text { protein } \\
\text { yield }\end{array}$ \\
\hline & $k g / h a$ & $\%$ & $\mathrm{~kg} / \mathrm{ha}$ & $k g / h a$ \\
\hline Maracay & $45,063 \mathrm{a}^{1}$ & $25.87 \mathrm{~d}$ & 11,675 a & 2,452 \\
\hline Florida-66 & $40,202 a b$ & $27.96 \mathrm{ad}$ & $11,231 \mathrm{ab}$ & 2,583 \\
\hline Certified Mesa Sirsa & $38,709 \mathrm{ac}$ & $26.49 \mathrm{~cd}$ & 10,277 ac & 2,261 \\
\hline Moapa & 34,431 bc & $28.15 \mathrm{ad}$ & 9,454 ad & 2,080 \\
\hline Hayden PX-1 & $34,392 \mathrm{bc}$ & $26.42 \mathrm{~cd}$ & $8,976 \mathrm{bd}$ & 1,974 \\
\hline Peruvian & $34,294 \mathrm{bc}$ & $26.96 \mathrm{~cd}$ & 9,099 bd & 2,002 \\
\hline UC-76-E & $31,894 \mathrm{bd}$ & $27.02 \mathrm{~cd}$ & $8,357 \mathrm{ce}$ & 1,839 \\
\hline Sonora-70 & $31,584 \mathrm{bd}$ & $28.02 \mathrm{ad}$ & $8,710 \mathrm{~cd}$ & 1,916 \\
\hline Certified Hayden & $31,270 \mathrm{bd}$ & $27.17 \mathrm{bd}$ & $8,477 \mathrm{ce}$ & 1,865 \\
\hline AZ Mexon (scald tol.) & 30,774 be & $28.46 \mathrm{ac}$ & 8,495 ce & 1,776 \\
\hline Mesa Sirsa Lateral Branch & 30,665 be & $26.23 \mathrm{~cd}$ & $7,908 \mathrm{cf}$ & 1,661 \\
\hline UC-Salton & 30,367 ce & $27.69 \mathrm{ad}$ & 8,273 ce & 1,820 \\
\hline UC-Cargo & 30,186 ce & $27.95 \mathrm{ad}$ & $7,988 \mathrm{cf}$ & 1,677 \\
\hline CUF-101 & $29,858 \mathrm{ce}$ & $27.65 \mathrm{ad}$ & $8,068 \mathrm{cf}$ & 1,775 \\
\hline WL-311 & $24,718 \mathrm{df}$ & 28.65 ac & $6,978 \mathrm{df}$ & 1,675 \\
\hline Liberty (NCW-20) & $22,772 \mathrm{df}$ & $29.84 \mathrm{a}$ & $6,209 \mathrm{dg}$ & 1,428 \\
\hline Thor & 21,631 ef & $28.38 \mathrm{ac}$ & $5,733 \mathrm{fg}$ & 1,374 \\
\hline Tanverde & $16,523 \mathrm{f}$ & $29.66 \mathrm{ab}$ & $4,673 \mathrm{~g}$ & 1,028 \\
\hline Mean & 31,074 & 27.70 & 8,366 & 1,844 \\
\hline
\end{tabular}

${ }^{1}$ Values in the same column followed by one or more letters in common do not differ significantly at the $5 \%$ probability level.

Tanverde. GF yields varied from a maximum of 45,063 for Maracay cultivar to a minimum of $16,523 \mathrm{~kg} / \mathrm{ha}$ for Tanverde cultivar.

The two variables GF yield and DM content were inversely related. The lowest GF yielder (Tanverde) presented the second highest DM content, while the highest GF yielder (Maracay) had the lowest DM content. Nevertheless, Maracay outyielded all other cultivars in DF, followed by Florida 66, Certified Mesa Sirsa, Moapa, Peruvian, and Hayden PX-1 in the same order as for GF yield, except for Peruvian and 
Hayden PX-1 cultivars. Maracay produced 4, 14, and 23\% more DF than Florida 66, Certified. Mesa Sirsa, and Moapa, respectively. Lowest DF yielders were Liberty (NCW-20), Thor, and Tanverde cultivars. In variety trials in the Dominican Republic (4) and Texas (6), Florida 66 was the top yielder. According to Ruelke (9), Florida 66 outyielded all other cultivars tested in Florida up to 1971.

The best DF yielders (Maracay, Florida 66, Certified Mesa Sirsa, Moapa, Peruvian, and Hayden PX-1) were also the best CP yielders, although Florida 66 replaced Venezuela at the top. CP yields ranged from 2,583 for Florida 66 to $1,028 \mathrm{~kg} / \mathrm{ha}$ for Tanverde.

Table 3 shows the data on DF yields per harvest. DF yields diminished at each successive harvest up to the sixth, because of a severe infestation of Phytophthora root rot. Similar yield reductions in different cultivars due to Phytophthora root rot were reported previously at the Lajas Valley by Freyre et al. (2). In the present study, it was observed that Maracay, Florida 66, and Certified Mesa Sirsa plots were the least affected by this disease; thus, these cultivars are more resistant to Phytophthora root rot.

Table 4 shows the mean results from the chemical analyses of the 18 cultivars. In general, a considerable degree of uniformity in chemical composition is noted among the different cultivars. A slight tendency can be observed for higher yielding cultivars to exhibit lower $\mathrm{CP}$ content and higher NDF content, although Florida 66 does not conform to this pattern. Table 5 shows simple correlation coefficients among the chemical constituents. As NDS and CP contents increased, NDF, ADF, H, and $\mathrm{C}$ contents decreased $(\mathrm{P}=.01)$. Contrary to expectations, Si content tended to decrease with an increase in the fibrous constituents: NDF ( $P$ $=.05)$ and $\mathrm{C}(\mathrm{P}=.01) . \mathrm{P}$ content also decreased with an increase in $\mathrm{C}$ content $(\mathrm{P}=.05)$ (table 5).

These results suggest that alfalfa production is feasible on the southern coast of Puerto Rico. However, in spite of being a perennial crop, alfalfa performs as an annual under most humid tropical conditions. As a result, alfalfa management is more complicated and laborious than grass management. Further research is needed to determine whether alfalfa cultivation for hay production is economical in Puerto Rico.

\section{RESUMEN}

Se realizó un estudio de 18 cultivares de alfalfa (Medicago sativa) en el Centro de Investigación y Desarrollo de Lajas para evaluar la aptitud y la producción en un periodo de 232 días (seis cortes del 31 de enero al 20 de septiembre de 1978). Los rendimientos de forraje seco variaron de 4,673 kg/ha (Tanverde—más bajo) a 11,675 kg/ha Maracay—más alto). Se determinó además que las mejores cultivares en rendimiento y persis- 
TABLE 3.-Dry forage yields of 18 alfalfa cultivars at 6 harvest dates over a 232-day period

\begin{tabular}{|c|c|c|c|c|c|c|c|}
\hline \multirow{2}{*}{ Cultivar } & \multicolumn{7}{|c|}{ Harvest dates ${ }^{1}$} \\
\hline & April 18 & May 18 & June 19 & July 13 & August 17 & September 20 & Total \\
\hline & & & & $\mathrm{kg} / \mathrm{ha}$ & & & \\
\hline Maracay & 2,993 & 1,988 & 2,170 & 1,586 & 1,732 & 1,206 & 11,675 \\
\hline Florida-66 & 3,338 & 1,589 & 2,114 & 1,434 & 1,888 & 868 & 11,231 \\
\hline Certified Mesa Sirsa & 2,719 & 1,608 & 1,721 & 1,298 & 1,202 & 1,729 & 10,277 \\
\hline Moapa & 3,167 & 1,650 & 2,027 & 1,533 & 1,077 & - & 9,454 \\
\hline Hayden PX-1 & 2,836 & 1,607 & 1,814 & 1,302 & 980 & 437 & 8,976 \\
\hline Peruvian & 3,021 & 1,782 & 1,966 & 1,229 & 946 & 155 & 9,099 \\
\hline UC-76-E & 2,469 & 1,820 & 1,870 & 1,273 & 868 & $57^{2}$ & 8,357 \\
\hline Sonora-70 & 2,716 & 1,317 & 1,731 & 1,316 & 968 & 662 & 8,710 \\
\hline Certified Hayden & 2,948 & 1,416 & 1,686 & 1,035 & 884 & 508 & 8,477 \\
\hline AZ Mexon (scald tol.) & 2,702 & 1,586 & 1,877 & 1,114 & 755 & 461 & 8,495 \\
\hline \multicolumn{8}{|l|}{ Mesa Sirsa } \\
\hline Lateral Branch & 2,252 & 1,289 & 1,447 & 981 & 734 & $401^{2}$ & 7,908 \\
\hline UC-Salton & 2,971 & 1,600 & 1,651 & 1,077 & 533 & 441 & 8,273 \\
\hline UC-Cargo & 2,668 & 1,486 & 1,553 & 922 & 789 & $570^{2}$ & 7,988 \\
\hline CUF-101 & 3,260 & 1,607 & 1,723 & 1,074 & 404 & - & 8,068 \\
\hline WL-311 & 3,071 & 1,445 & 1,556 & 745 & 161 & - & 6,978 \\
\hline Liberty (NCW-20) & 2,691 & 1,347 & 1,276 & 718 & 177 & 一 & 6,209 \\
\hline Thor & 2,394 & 1,074 & 1,116 & 690 & 274 & $185^{3}$ & 5,733 \\
\hline Tanverde & 968 & 700 & 1,021 & 671 & 586 & $727^{2}$ & 4,673 \\
\hline Mean & 2,732 & 1,495 & 1,684 & 1,111 & 831 & 467 & 8,366 \\
\hline
\end{tabular}

${ }^{1}$ Each yield is a mean of 4 replicates.

${ }^{2}$ Mean of 3 replicates.

${ }^{3}$ Mean of 2 replicates. 
TABLE 4.-Mean chemical and mineral composition of 18 alfalfa cultivars during a 232-day period

\begin{tabular}{|c|c|c|c|c|c|c|c|c|c|c|}
\hline Cultivar & $\mathrm{NDF}^{1}$ & NDS & ADF & $\mathrm{H}$ & $\mathrm{L}$ & $\mathrm{C}$ & $\mathrm{Si}$ & $\mathrm{CP}$ & $\mathrm{P}$ & $\mathrm{K}$ \\
\hline \multicolumn{11}{|c|}{$\%$} \\
\hline Maracay & 35.37 & 64.63 & 30.13 & 5.24 & 9.87 & 18.90 & .93 & 21.38 & .33 & 2.04 \\
\hline Florida- 66 & 33.37 & 66.63 & 28.60 & 4.77 & 9.43 & 16.64 & 1.68 & 22.81 & .34 & 2.05 \\
\hline Certified Mesa Sirsa & 36.14 & 63.86 & 28.73 & 7.41 & 10.05 & 17.31 & 1.14 & 21.50 & .35 & 2.01 \\
\hline Moapa & 34.49 & 65.51 & 28.84 & 5.65 & 9.10 & 18.17 & 1.12 & 21.50 & .34 & 2.18 \\
\hline Hayden PX-1 & 34.91 & 65.09 & 29.71 & 5.20 & 10.03 & 18.08 & 1.00 & 20.50 & .36 & 2.24 \\
\hline Peruvian & 35.21 & 64.79 & 29.06 & 6.15 & 10.95 & 16.71 & .98 & 21.88 & .38 & 2.14 \\
\hline UC-76-E & 34.22 & 65.78 & 28.63 & 5.59 & 10.00 & 16.82 & 1.66 & 21.75 & .35 & 1.92 \\
\hline Sonora-70 & 36.18 & 63.82 & 29.69 & 6.49 & 10.33 & 17.63 & 1.38 & 21.50 & .36 & 1.92 \\
\hline Certified Hayden & 35.31 & 64.69 & 29.22 & 6.09 & 10.07 & 17.60 & .84 & 21.81 & .35 & 2.14 \\
\hline AZ Mexon (scald tol.) & 36.70 & 63.30 & 31.08 & 5.62 & 12.46 & 17.03 & 1.31 & 21.13 & .36 & 2.16 \\
\hline \multicolumn{11}{|l|}{ Mesa Sirsa } \\
\hline Lateral Branch & 36.85 & 63.15 & 30.34 & 6.51 & 10.36 & 18.06 & 1.30 & 20.88 & .36 & 1.90 \\
\hline UC-Salton & 34.78 & 65.22 & 29.00 & 5.78 & 10.46 & 17.12 & 1.40 & 21.69 & .37 & 2.17 \\
\hline UC-Cargo & 35.80 & 64.20 & 29.65 & 6.15 & 10.28 & 17.28 & 1.85 & 21.25 & .36 & 2.12 \\
\hline CUF-101 & 35.50 & 64.50 & 29.83 & 5.67 & 10.75 & 17.61 & 1.34 & 21.81 & .35 & 2.00 \\
\hline WL-301 & 33.74 & 66.26 & 28.88 & 4.86 & 10.39 & 15.92 & 2.35 & 23.50 & .37 & 2.07 \\
\hline Liberty (NCW-20) & 32.53 & 67.47 & 27.29 & 5.24 & 9.18 & 15.90 & 1.48 & 23.31 & .37 & 2.05 \\
\hline Thor & 30.76 & 69.24 & 26.73 & 4.03 & 10.20 & 13.76 & 2.06 & 23.56 & .37 & 2.17 \\
\hline Tanverde & 35.54 & 64.46 & 28.96 & 6.58 & 10.04 & 16.90 & 1.70 & 21.75 & .35 & 2.12 \\
\hline Mean & 34.86 & 65.14 & 29.13 & 5.73 & 10.22 & 17.08 & 1.42 & 21.86 & .36 & 2.08 \\
\hline
\end{tabular}

${ }^{1}$ NDF, neutral-detergent fiber; NDS, neutral-detergent solubles; ADF, acid-detergent fiber; $\mathrm{H}$, hemicellulose; L, lignin; C, cellulose; Si, silica; $\mathrm{CP}$, crude protein; $\mathrm{P}$, phosphorus; $\mathrm{K}$, potassium. 
TABLE 5.- Simple correlations among constituents of 18 alfalfa cultivars

\begin{tabular}{|c|c|c|c|c|c|c|c|c|c|c|}
\hline Constituent & NDF & NDS & $\mathrm{ADF}$ & $\mathrm{H}$ & $\mathrm{L}$ & C & $\mathrm{Si}$ & $\mathrm{CP}$ & $P$ & $\mathrm{~K}$ \\
\hline $\mathrm{DM}^{1}$ & -.41 & .41 & -.43 & -.23 & -.06 & $-.56^{* 2}$ & $.57^{*}$ & $.58^{*}$ & .28 & .20 \\
\hline $\mathrm{NDF}$ & & $-1.00^{* * 3}$ & $.88^{* *}$ & $.79^{* *}$ & .45 & $.77^{* *}$ & $-.50^{*}$ & $-.82^{* *}$ & -.22 & -.25 \\
\hline NDS & & & $-.88^{* *}$ & $-.79^{* *}$ & -.45 & $-.77^{* *}$ & $.50^{*}$ & $.82^{* *}$ & .22 & .25 \\
\hline $\mathrm{ADF}$ & & & & .40 & $.57^{*}$ & $.75^{* *}$ & -.42 & $-.78^{* *}$ & -.26 & -.12 \\
\hline $\mathrm{H}$ & & & & & .13 & $.51^{*}$ & -.42 & $-.59^{* *}$ & -.08 & -.33 \\
\hline $\mathrm{L}$ & & & & & & -.04 & -.02 & -.27 & .37 & .09 \\
\hline $\mathrm{C}$ & & & & & & & $-.70^{* *}$ & $-.82^{* * *}$ & $-.57^{*}$ & -.17 \\
\hline $\mathrm{Si}$ & & & & & & & & $.64^{* *}$ & .30 & -.10 \\
\hline $\mathrm{CP}$ & & & & & & & & & .29 & .00 \\
\hline $\mathrm{P}$ & & & & & & & & & & .20 \\
\hline
\end{tabular}

${ }^{1} \mathrm{DM}$, dry matter; NDF, neutral-detergent fiber; NDS, neutral-detergent solubles; ADF, acid-detergent fiber; H, hemicellulose; L, lignin; C, cellulose; Si, silica; CP, crude protein; P, phosphorus; K, potassium.

${ }^{2}$ Significant at the 5-percent level.

${ }^{3}$ Significant at the 1-percent level. 
tencia fueron Florida 66, Moapa, Certified Mesa Sirsa, Peruvian y Hayden PX-1. Debido principalmente a la alta incidencia de la pudrición de la corona, causada por el hongo Phytophthora, el rendimiento de forraje seco bajó notablemente después del quinto corte en toda las cultivares, excepto en Maracay, Florida 66 y Certified Mesa Sirsa. Los promedios globales de fibra neutrodetergente, fibra ácidodetergente, lignina, celulosa, sílice, proteína bruta, fósforo y potasio en las 18 cultivares fueron 34.86, 29.13, $10.22,17.08,1.42,21.86, .36$ y $2.08 \%$, respectivamente. Los insectos no causaron gran daño en este estudio.

\section{LITERATURE CITED}

1. Duncan, D. B., 1955. Multiple range and multiple F tests, Biometrics 11:1-42.

2. Freyre, R. H., Rico-Ballester, M., Vázquez-Romero, R., and Warmke, H. E., 1954. Alfalfa yields declined between the fifth and eighth harvest, USDA Exp. Stn. Ann. Prog. Rep.

3. Goering, H. K. and Van Soest, P. J., 1970. Forage fiber analyses (apparatus, reagents, procedures and some applications), USDA Agric. Handbook 379.

4. Gutiérrez de Flores, T., Cuevas-Pérez, F., Arroyo-Aguilú, J. A., Wagner, B., Peterson, R. A., and Santhirasegaram, K., 1977. Adaptación, comportamiento y producción de alfalfa (Medicago sativa) en República Dominicana. Presented at the 8th Panamerican Congress of Veterinary Medicine and Zootechnics, Santo Domingo, Dominican Republic, August 1-6.

5. Hine, R. B., Gray, F. A., and Schonhorst, M. H., 1972. Phytophthora root rot of alfalfa in Arizona, Plant Dis. Rep. 56:472-3.

6. Holt, E. C., 1971. Evaluation of alfalfa varieties for hay production, Texas Agric. Exp. Stn. Rep. 3481, College Station, Texas.

7. Riera, A., 1946. A laboratory recommendation of lime to an acid soil checks with expected pH changes, J. Agric. Univ. P. R. 30 (3):184-5.

8. - 1955 . The method of foliar diagnosis as applied to sugarcane, II. The chemical analyzes of sugarcane-leaf samples, Univ. P. R. Agric. Exp. Stn. Bull. 123.

9. Ruelke, O. C., 1974. Yield, quality and potential of "Florida 66" alfalfa, Presented at the 23rd Annual Beef Cattle Course, Univ. Fla., Gainesville, Fla., May 4.

10. Snedecor, G. W. and Cochran, W. C., 1967. Statistical Methods, 6th ed., The Iowa State Univ. Press, Ames, Iowa.

11. Vélez-Santiago, J., 1977. Univ. P. R. Agric. Exp. Stn. Prog. Rep. 